\title{
Information persistence in the integration of partial cues for object recognition
}

\author{
ERNEST GREene \\ University of Southern California, Los Angeles, California
}

\begin{abstract}
A great many studies have shown that the perceptual effects of very brief visual stimuli can persist beyond the duration of the stimulus itself. These effects include sustained perception of the stimulus even though it is no longer present and the integration of information across an interstimulus interval. These two forms of sustained activity can be characterized as visible persistence and information persistence. Iconic memory protocols and a number of discrimination tasks have demonstrated the existence of information persistence that can last up to several hundred milliseconds, but there is little evidence that the cues needed for identification of objects can be transferred across intervals in this range. In the present experiments, a minimal transient discrete cue protocol was used to demonstrate that shape cues, these being provided by subsets of dots that mark the outer boundary of nameable objects, can be integrated over several hundred milliseconds and that the duration is a function of ambient room illumination. The experiments further evaluated whether this information persistence is mediated by visible persistence. Although both perceptual effects have durations that are an inverse function of room illumination, the ability to integrate partial shape cues was not determined by the duration of visible persistence.
\end{abstract}

At the retina, a brief pulse of light causes a response that rises rapidly and decays slowly, outlasting the flash. (Adrian \& Matthews, 1927)

There is abundant evidence that the information provided by sensory signals is preserved for an interval that may be far longer than the stimulation itself. Thus, even for the briefest of stimuli, there is some form of sustained neural response that provides for integration of information being generated by successive events. In vision, this process has been described using various terms, including visual persistence (see Long, 1980), visible persistence (see Coltheart, 1980), visual information store (Sperling, 1960), iconic memory (Neisser, 1967), and short-term visual storage (Haber \& Standing, 1969).

What is most pertinent to the present work are the methods introduced by Eriksen and Collins $(1967,1968)$. These investigators briefly displayed two dot patterns in rapid succession. Dot placement in the two patterns was complementary, so that a composite of the two would allow a three-consonant trigram to be seen if the two were displayed quickly enough. It was considered likely that persistence allowed stimulus information from the first pattern to be carried forward across the interval, making it possible for that information to be combined with the stimulus pattern that followed about $100 \mathrm{msec}$ later. The results of Eriksen and Collins $(1967,1968)$ appeared to be congruent with earlier work by Sperling (1960) and Averback and Coriell (1961), each of whom had suggested some form of sustained sensory trace as part of the normal mechanism for storing stimulus information.
However, the question of whether sustained activity is a prerequisite for accessing long-term memory stores has not been adequately addressed, nor has it been shown that the information needed for recognition can be integrated over intervals in the 100-msec range. The recognition required by Eriksen and Collins $(1967,1968)$ was from a very small store of overlearned letter trigrams. The task might well be accomplished by relatively narrow discrimination criteria that have little to do with normal shape and object recognition. Furthermore, subsequent studies making use of the successive-display paradigm have employed discrimination tasks that do not address the possible role for visible persistence in the retrieval of long-term memories, (see Di Lollo, 1977, 1980; Phillips, 1974; Sakitt \& Long, 1978, 1979).

As a related issue, a number of experimentalists and theorists have argued that the sustained neural response that mediates integration of information is also experienced as visible persistence, wherein the stimulus is consciously perceived as being present for a period that can be far longer than its actual duration. One might hypothesize that the process of probing memory stores to achieve recognition requires sustained sensory activity, and this could provide a raison d'être for the phenomenon of visible persistence.

Coltheart (1980) provided an especially thorough review of this issue and formulated a clear distinction between two kinds of sustained perceptual activity: visible persistence, which provides a conscious experience, as though the stimulus were still present, and information persistence, in which the traces of the stimulus are not consciously perceived. The Eriksen and Collins (1967,

E. Greene, egreene@usc.edu 
1968) protocol was cited as requiring transfer of information, which may not (or might not) be mediated by visible persistence. Given this ambiguity, a second goal was to examine the question of whether visible persistence mediates the temporal integration of partial shape cues.

A third goal was to determine the effects of lighting on visible and information persistence, since the interval over which the stimulus trace persists is thought to change as a function of the relative intensity of the stimulus, which in turn could determine the duration over which information can be integrated. Studies have shown divergent effects, with some showing an increase in duration of persistence with a decrease in stimulus intensity and others showing an increase in duration as stimulus intensity is increased. These can be described as inverse-intensity and directintensity effects, respectively.

Efron (1970) found inverse-intensity effects by having subjects synchronize the onset of a click to match the perceived offset of a brief flash and found that the duration of the persistence increased with decreasing flash intensity. Bowen, Pola, and Matin (1974) reported similar effects when subjects were asked to make the synchrony judgment using a spatially separated flash. Hansteen (1971) reached the same result, using a different experimental method.

Conversely, Sakitt (1976a, 1976b) found a directintensity effect when subjects were asked to synchronize the onset of a click with the disappearance of a letter array. Keele and Chase (1967) used a partial report protocol in which a circular array of 10 alphanumeric characters was displayed briefly at several levels of intensity, followed by a probe cue with an interstimulus interval that varied between 0 and 5,000 msec. Accuracy of report of the designated character was best at the highest level of stimulus intensity and poorest at the lowest level of intensity, meaning that the increased intensity of the stimulus prolonged the duration of information persistence. Similar direct effects have been found by Di Lollo and Woods (1981), Long and McCarthy (1982), Long and Sakitt (1980), and Sakitt and Long (1978).

Hawkins and Shulman (1979) suggested that the inverseversus direct-intensity effects can be ascribed to two different task demands: one that marks the time until the first perceived decrement in intensity of the stimulus, and another that reflects the full duration of the stimulus trace. Type I persistence, which manifests an inverse-intensity effect, is seen when subjects judge the initial decline in intensity of the stimulus, as they are likely to do when asked to set the onset of a probe marker to the offset of the stimulus. Type II persistence, which manifests a direct-intensity effect, is found when the subject is asked to mark the disappearance of the stimulus or report information that may be present until the stimulus has completely disappeared. The duration of the full trace determines the degree to which stimulus information survives across time and, thus, can contribute to discrimination judgments. Long (1979), Long and Gildea (1981), and Nisly and Wasserman (1989) have argued along similar lines.

Di Lollo and Bischof (1995) have provided an especially useful review of the relevant literature, adding con- siderable attention to differences in the level of ambient illumination that was present during testing. They argued that the level of background illumination determines whether one is dealing with photopic versus scotopic vision and this, in turn, affects whether one sees an inverseintensity effect. In general, experiments that used dim to dark ambient levels yielded inverse-intensity effects, and those that showed no intensity effects used bright room illumination.

Background intensity, however, does not fully explain the results from studies that have shown positive intensity effects on persistence. Here, Di Lollo and Bischof (1995) proposed that differences in stimulus intensity affect the visibility of the stimulus - that is, salience - which alters performance on the task by means other than the duration of visible persistence. They argued that the key evidence for differences in salience is to be found in the level of performance when there is no delay in the presentation of stimulus elements. For example, in Keele and Chase's (1967) study, the accuracy of partial report was best at the highest level of stimulus intensity and poorest at the lowest level of intensity. However, performance was superior even when the delay was zero, suggesting that visibility of the stimulus, rather than persistence of information, was the basis for the stimulus intensity effect. The same explanation can be offered for the positive stimulus intensity effects reported by Di Lollo and Woods (1981), Long and McCarthy (1982), Long and Sakitt (1980), Sakitt (1976a, 1976b), and Sakitt and Long (1978).

Di Lollo and Bischof (1995) made a convincing case that inverse-intensity effects can be ascribed to visible persistence per se and that direct-intensity effects are due to differences in perceptibility (salience). According to their analysis, testing subjects under photopic conditions (i.e., with the room well lighted) should provide relatively short durations of visible persistence, and testing them under scotopic conditions (i.e., with the room dark) should produce relatively long durations. One of the major treatment variables of the present work, therefore, was manipulation of room lighting.

With respect to information persistence, however, Di Lollo and Bischof (1995) argued that salience of the stimulus is the major factor in specifying the duration over which information can be integrated. If the salience of the stimulus is about the same for the two room illumination conditions, there should be no differential influence on the duration of information persistence. For the present work, the levels of stimulus intensity were chosen with the goal of providing equal salience, so one would not expect any effect of stimulus intensity or room illumination. Coltheart (1980) seems to have made the same prediction, saying that reduced stimulus intensity should yield longer durations of visible persistence but should have no influence on information persistence.

Each of the methods that have been used to assess visible persistence has failings. Di Lollo and Bischof (1995) criticized the use of synchrony-of-offset judgments as the basis for specifying the duration of persistence. An example of a synchrony judgment would be a case in which a brief stimulus is presented, this being perceived as hav- 
ing a duration that is longer than the stimulus itself, and the subject must adjust the onset of a probe marker (e.g., a click), so that the onset of the probe is placed at the perceived offset of the stimulus. Di Lollo and Bischof faulted this method as being difficult to implement and for yielding variable results, regardless of sensory modality, and for giving results that can differ according to the content of the stimulus being evaluated. This echoes similar criticism by Irwin and Yeomans (1986), who described the method as "unreliable" (see also Allan, 1979; Turvey, 1978).

On the other hand, the methods developed by Hogben and Di Lollo (1974) may assess performance demands, rather than (or more than) visible persistence. They presented subjects with two subsets of dots (12 dots in each subset), with the first subset occupying randomly selected locations in a $5 \times 5$ matrix of possible positions and the second subset occupying other locations. In aggregate, the 24 dots leave one cell of the matrix unoccupied, and the subjects' task is to specify which cell was left empty by the display of the two subsets. To use this method to assess the duration of persistence, one adds a delay between the brief displays of the first and second dot subsets. The subjects' accuracy at judging the location of the empty cell declines as that interval increases, commonly reaching chance levels at 100-200 msec (depending on stimulus and background light levels, as has been outlined above).

A problem with this protocol is that the measure is determined partly by the discrimination to be accomplished. A $5 \times 5$ array was chosen because it was the size that would allow for $100 \%$ performance if 24 of the elements were presented simultaneously; use of a larger array would reduce the accuracy with which the empty cell could be identified. Thus, array size itself provides a demand on discrimination, and when the subject is faced with a requirement to locate an empty cell on the basis of a fading iconic trace, it is not clear how much the $5 \times 5$ array size might contribute to the estimate of persistence. Without laboring the point, this and similar procedures not only reflect visible persistence per se, but also are loaded by the particular discrimination that must be accomplished.

An additional problem in the interpretation of the persistence literature is the fact that there is evidence that some reports of persistence duration could have been a result of residual emission from the display itself, rather than of the persistence of a perceptual trace. Rayner and Pollatsek (1983) were interested in examining persistence of information that might survive execution of saccadic eye movements, but their results have implications for whether one can rely on other reports that deal with the duration of persistence.

Using the $5 \times 5$ array method of Hogben and Di Lollo (1974) and essentially replicating an earlier experiment done by Jonides, Irwin, and Yantis (1982), Rayner and Pollatsek (1983) found that a substantial amount of information is transferred across a $40-\mathrm{msec}$ interstimulus interval during which a saccade is executed. However, one can infer that the perceptual system sustains information across the interstimulus interval only if the stimulus itself is not still present. For that reason, in many, or per- haps most, of the studies of visible persistence, cathode ray tubes with short-lived phosphors (either P-4 or P-31) have been used for display of the stimuli. These phosphors are known to have fast decay rates, which generally fall to about $1 \%$ of maximum brightness in microseconds. Rayner and Pollatsek (1983) noted that this 1\% residual persists for a second or more, so they included additional control measures. The control results indicated that the ability to judge the location of the empty cell was made possible through direct perception of this afterglow.

As further evidence that transfer across the interstimulus interval (and across a saccade) is mediated by residual phosphor emission, Irwin, Yantis, and Jonides (1983) and Jonides, Irwin, and Yantis (1983) failed to replicate Jonides et al.'s (1982) report using a $5 \times 5$ array of lightemitting diodes (LEDs) that had ultrafast rise and fall of emission and for which there was no residual afterglow. They found no evidence that information could be integrated across the time interval during which the saccade was executed. These results again argue that the initial information persistence that was reported by Jonides et al. (1982) was based on residual afterglow of phosphors. Other phosphor trace problems have been reported by Di Lollo, Enns, Yantis, and Dechief (2000).

Di Lollo, Seiffert, Burchett, Rabeeh, and Ruman (1997) evaluated four phosphors that are commonly used for psychophysical research and found residual afterglow to be a problem for $\mathrm{P} 4, \mathrm{P} 31$, and $\mathrm{P} 46$, but not for the $\mathrm{P} 15$ phosphor. Nonetheless, for the present work, it was deemed prudent to use LEDs for stimulus display in order to avoid any possibility that recognition might be drawing on residual stimulus afterglow. In the present research, a $64 \times 64$ LED array was used, along with microprocessor control and custom software, which provided for submillisecond control of stimulus emission, along with assurance that there was no residual emission.

Furthermore, being mindful of the cautions given by Irwin and Yeomans (1986) and Di Lollo and Bischof (1995) that synchrony judgments are unreliable, another methodfirst suggested by Newton (1730/1952) — was adopted for assessing visible persistence. Details for this protocol will be given in the Method section, and further background will be provided in the General Discussion section.

As the briefest of previews, the present research evaluated the integration of cues needed for object recognition, using a method that was similar in concept to the successive-display paradigm developed by Eriksen and Collins $(1967,1968)$. In the two experiments, a minimal transient discrete cue (MTDC) protocol was used, which called for naming of objects, each being shown only as a spaced sampling of dots that marked the outer boundary of the object. This set of dots was further broken into two subsets, each having a number of dots too small to elicit recognition of the object. These subsets were then displayed with several levels of temporal separation, to determine what proportion of objects could be named at the various time intervals. These tests were conducted under several levels of room illumination, and these conditions provided a major influence on the duration on persistence, as described above. 
The results show that shape cue information can be integrated across intervals in that range upward of several hundred milliseconds, with the duration of the information persistence being a function of room illumination. The minimal, transient shape cues can be integrated over a longer interval if the room is dim, and longer yet if the room is dark. Although visible persistence also differed for the two levels of room illumination, with persistence being longer in the darkened room, the duration of the visible persistence did not explain the differences in recognition.

\section{EXPERIMENT 1}

\section{Method}

Subjects. Ten University of Southern California undergraduates served as subjects in Experiment 1. Each was tested and found to have normal or corrected-to-normal vision. Each was provided with a description of the general task conditions but was not told anything about the goals of the experiment. The subjects were paid for their participation.

Apparatus. Visible persistence and shape recognition tests were conducted with the stimuli being presented on a $64 \times 64$ LED array, which is designated as the display board. The board was constructed from Marktech modules, each element of the module being a GaAlAs LED that emitted with a wavelength of $660 \mathrm{~nm}$ (red). The LEDs had a rise-and-fall time in the range of 50-100 nanosec. Luminous intensity will be described below.

Each LED of the display board had a diameter of $5.08 \mathrm{~mm}$ and a center-to-center spacing of $7.62 \mathrm{~mm}$. The span of the entire array was $48 \mathrm{~cm}$, measured between the centers of the outer rows (or columns) of LEDs. The display board was attached to the wall at a distance of $3.53 \mathrm{~m}$ from the subject and at a height that was approximately $10^{\circ}$ above eye level. Given the fixed viewing distance, the LED dimensions can be expressed in minutes and degrees of visual angle. In these units, the diameter of each LED was $4.95 \mathrm{~min}$ of arc, center-to-center spacing was $7.42 \mathrm{~min}$ of arc, and the horizontal and vertical dimensions of the full LED array was $7.74^{\circ} \times 7.74^{\circ}$ of arc.

The LEDs of the display board were connected so that a given LED could be turned on - that is, lighted - by applying power to a given row and column of the array. The rows and columns were serviced by decoders and drivers that applied power to the designated row and column upon receipt of an $x, y$ address. The presentation of a sequence of $x, y$ addresses was controlled by a USBSimm (J. Gordon) that ran at a clock speed of $24 \mathrm{MHz}$. This simm received the table of addresses to be displayed and the time intervals to be used in the displays from a Mac G4 Cube, which also served as the interface for experimental control and recording of subject data. Stimulus display, experimental control, and data recording were effected using custom software and firmware.

Treatment A: Luminance levels. As was outlined in the introduction, one goal of Experiment 1 was to use testing and stimulus conditions that would create differentials in visible persistence. A number of studies have shown that the duration of visible persistence is longer in the dark than in light and is inversely related to the brightness of the stimulus (see Coltheart, 1980; Di Lollo \& Bischof, 1995; Long, 1980). Therefore, the testing of subjects, both for the pretest qualification of shapes (see the Qualifying the Shapes section) and for Experiment 1 (see the Treatment B section) was done with the room lighted or darkened and using a different level of LED emission for each of these ambient illumination conditions.

Where testing was to be done with the room lighted, illumination was provided by a single 4 -ft fluorescent tube, set within a recessed ceiling fixture that had a plastic diffuser. Average luminance from the wall that faced the subject was $25 \mathrm{~cd} / \mathrm{m}^{2}$.

For lighted room testing, mean reflectance from nonemitting LEDs was $20 \mathrm{~cd} / \mathrm{m}^{2}$, and the epoxy matrix that surrounded each LED had a mean reflectance of $9 \mathrm{~cd} / \mathrm{m}^{2}$. Emission from activated
LEDs had a mean of $96 \mathrm{~cd} / \mathrm{m}^{2}$. The emission measures were taken using an ultrafast photodiode, the readings from which were then calibrated using a photometer from Quantum Instruments.

For testing with the room darkened, the only source of light was from the flat screen monitor of the Mac computer, set to the lowest brightness level. The subject could not see the face of the monitor, but the screen did provide dim illumination of the wall on one side of the subject. The reflected luminance of the display board and of the wall on which it was mounted was below the sensitivity range of the photometer and can be considered to be functionally zero.

LED emission for testing with the room darkened was reduced to enhance the duration of visible persistence, with the luminance of each pulse being $9.6 \mathrm{~cd} / \mathrm{m}^{2}$.

Shape stimuli. The shapes to be identified were nameable objects that were originally derived from the Macmillan Visual Dictionary or from Hemera's PhotoObjects Vol. 1. Each shape had been rendered as a string of dots that traced the outer boundary, as is illustrated in the upper panel of Figure 1. The dot sequence did not include any internal contour. In addition, the rules for dot placement required that one be able to move from dot to dot in a complete circuit around the shape, without returning to any dot already visited until completion of the circuit.

Each dot was positioned for display by the $64 \times 64$ LED array and, thus, could be specified by an $x, y$ address. The aggregate set of addresses for a given shape is designated as an address table. Each shape was sized so that it would span the full dimension of the array in either the horizontal or the vertical direction.

Ninety-six shapes were used for this experiment. They are listed in Table 1 along with (1) the number of dots in the perimeter of each shape, (2) the area enclosed by the perimeter (as a count of internal dots and including the perimeter dots themselves), and (3) the difficulty index, as detailed below. These shapes were chosen from a much larger set on the basis of judgments made by a separate group of subjects, having the goals of culling those shapes that could not be reliably identified and providing a difficulty index for each shape.

Qualifying the shapes. For the shape recognition portion of Experiment 1 , each shape was to be displayed for a very brief interval, using only a percentage of the total inventory of border dots (designated as the display set). Both of these stimulus conditions will be detailed below in the Specifying the Display Set Using the Difficulty Index and the Treatment B sections. The size of the display set for a given shape was determined through preliminary testing of a larger set of candidate shapes by two additional groups of subjects. Six of these subjects judged the candidate set in a lighted room, and 6 other subjects judged the candidate set in a darkened room.

The presentation of a given evenly spaced display set was very brief, with onset of successive dots coming immediately upon offset of the preceding dot. Display of a 5\% sample from a boundary that contained 200 dots would take only $1 \mathrm{msec}$. Even where the percentage of dots to be displayed was substantially higher, the full sample could be presented with no perceptible movement artifact and a minimal sense of time differentials.

The subject was first shown each candidate shape, using a 5\% sample from the inventory of boundary dots, with the requirement that the sample be spaced and briefly presented as detailed in the Specifying the Display Set Using the Difficulty Index and the Treatment B sections. After each shape had been shown, the subject was encouraged to name the shape or make a guess. The subject was not told whether or not the response was correct. If the shape was correctly named, the size of the sample was recorded, and the shape was removed from the list. For those shapes that could not be identified, the percentage was increased by $5 \%$ - that is, to $10 \%$ on this iteration - and these shapes were again displayed. With each iteration, another $5 \%$ was added.

Any shape for which recognition by any subject required more than $50 \%$ of the full complement of dots was excluded from the subsequent experiments. In other words, if any subject required more than a $50 \%$ display set, the shape was rejected. This procedure yielded the 96 shapes that were used in Experiment 1. 

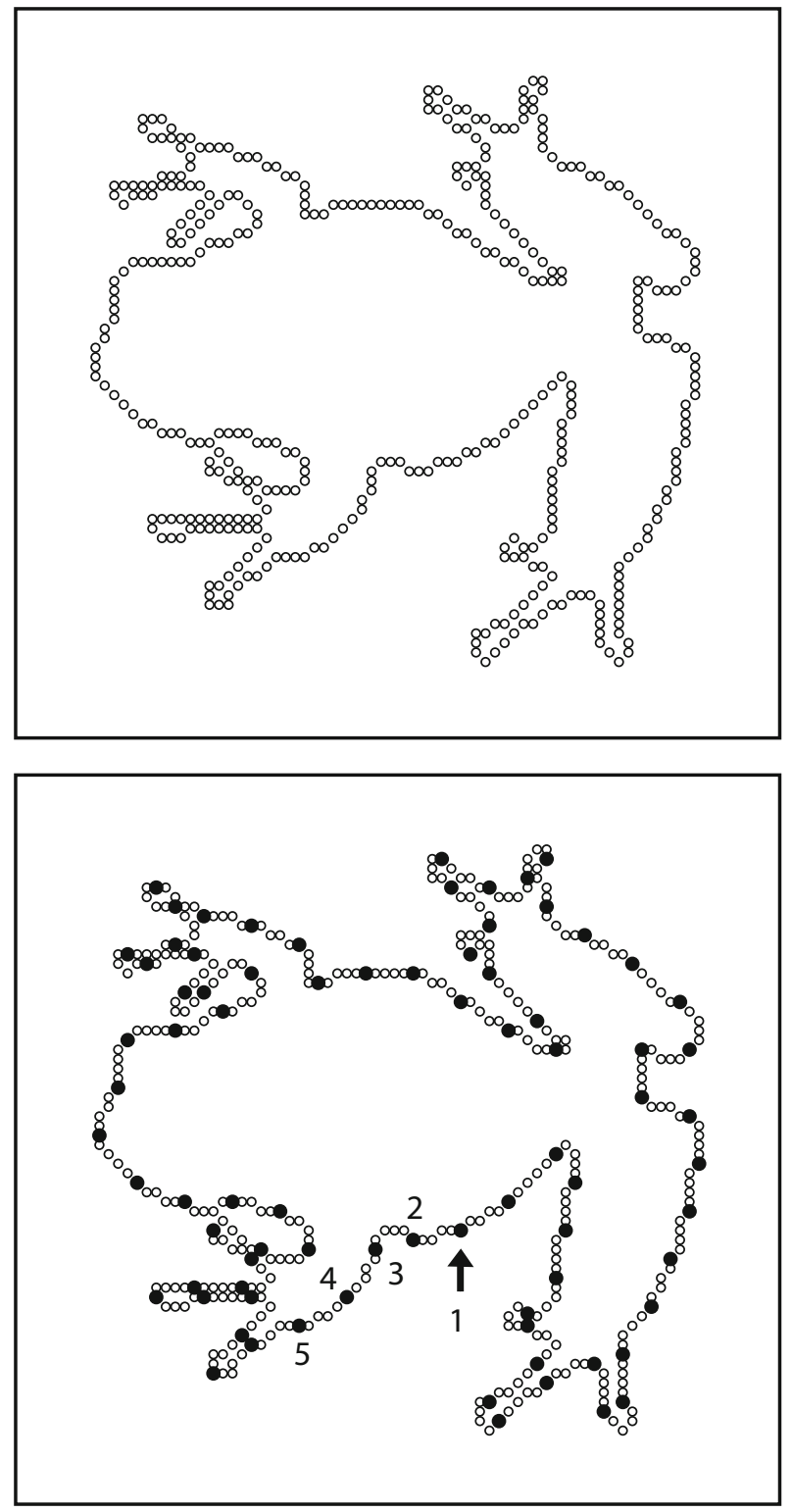

Figure 1. Top: Full complement of boundary dots for one of the shapes used in the two experiments. Bottom: Example of a display set, these dots being specified using filled circles that have been resized for purposes of illustration. The size of the display set was determined by the difficulty index (see the Qualifying the Shapes section). The specific dots to be shown on any given trial was determined by selecting a starting point at random (designated with an arrow), then counting clockwise and selecting every $n$th position, as needed, to specify the number of dots in the display set. Note that the display set was further divided into subsets that were displayed with time differentials (see the Treatment B section). The composition of the subsets was determined by whether the dot was at an odd or an even location in the counting sequence. In the bottom panel, the first five dots in the display set have been numbered. Dots 1,3 , and 5 would begin the sequence for the odd subset; Dots 2 and 4 would be the first two dots in the even subset.

On the basis of the percentage of dots required for recognition and the standard deviation for this value across subjects, it was possible to derive a difficulty index for each shape or, rather, a difficulty index at each level of room illumination (with corresponding dif- ferentials of LED intensity, as described in the Treatment A: Luminance Levels section). For each of the illumination conditions, the 75 th percentile of successful recognitions across subjects was determined for each shape. This value specified the sample percentage that should provide for successful recognition of a given shape on approximately $75 \%$ of the trials on which that shape was displayed. The index provided a means of equating the shapes, in that a display of each shape using the percentage of dots indicated by the index should allow approximately the same hit rate for the full inventory of shapes. The averages at the 75 th percentile were significantly different as a function of room illumination, but the magnitude of the difference was only $2 \%$. Given this small difference, and the fact that subsequent experimental testing of a given subject would be done under both of the room illumination conditions, the average of the 75 th percentiles for lighted and darkened room tests was used as the difficulty index for the following experiments. The difficulty index for each of the 96 shapes is shown in Table 1.

To recap, the value of the difficulty index for a given shape can be used to determine what percentage of dots to display. This percentage should allow recognition of the shape by approximately three fourths of the subjects if all the dots in the display set were shown for a very brief moment.

Specifying the display set using the difficulty index. The difficulty index not only controlled for differences in difficulty across shapes, but also eliminated redundant dots that would create a floor at which very few shapes would fail to be recognized. The number of dots in the display set is the percentage specified by the difficulty index, multiplied by the total number of border dots (rounding as required by spacing constraints)

For any given presentation of a shape, the specific dots to be used for the display set were selected by choosing a random starting position at the border and then counting clockwise around the border, including every $n$th dot, as needed, to have the final number of selected positions match the number of dots in the set to be displayed. This is illustrated in the bottom panel of Figure 1. Depending on the number of dots in the border and in the display set, the final step in completing the circuit would often be smaller than $n$, which was deemed to be of no practical consequence.

Treatment B: Differential T3 interval between odd and even dot subsets. In the Qualifying the Shapes section (for testing candidate shapes), the timing of the dot display was described as being very brief. It is appropriate now to be more specific. For testing the candidate shapes and for the following experiments, each dot was shown with a pulse width of $0.1 \mathrm{msec}$ and with pulse separationthat is, the onset-to-onset interval for display of successive dotsalso being $0.1 \mathrm{msec}$. Pulse width and pulse separation are designated as T1 and T2, as illustrated in panels A and B of Figure 2.

If all the display set dots were shown with these time intervals, they would appear to be displayed at virtually the same moment. However, the dots to be displayed for a given shape were divided into odd and even subsets that were displayed with the addition of a third time interval, designated as T3. For this purpose, the dots in the full display set are sequentially numbered, with the odd-numbered dots being assigned to one subset and the even-numbered dots to a second subset, as is illustrated in the bottom panel of Figure 1.

The T3 interval specifies the time between offset of the first subset and onset of the second. In Experiment 1, four levels of T3 were used - namely, $0,30,90$, and $270 \mathrm{msec}$, as is illustrated in panel C of Figure 2. For each subject, a given shape was assigned to only one of these treatment levels, as will be described more fully below.

One would not expect very many shapes to be recognized on the basis of information provided by display of the first subset. On the basis of the distributions provided by subjects who qualified the shapes (section 2.5), it can be estimated that the hit rate would be no more than $14 \%$. In addition, subsequent work from this lab has examined the level of recognition by 10 subjects when just one of the subsets was briefly presented twice. T1, T2, and T3 were equal to $0.1,0.1$, and $45 \mathrm{msec}$, respectively, and the average hit rate was $19 \%$. 
Table 1

Names of the Objects (Shapes) Tested in the Two Experiments, Number of Dots in the Perimeter, Number of Dots That Would Fill the Area Contained by the Border, and Difficulty Index

\begin{tabular}{|c|c|c|c|c|c|c|c|c|c|}
\hline Shape No. & Shape Name & $\begin{array}{l}\text { Dots in } \\
\text { Perimeter }\end{array}$ & $\begin{array}{c}\text { Dots in } \\
\text { Area }\end{array}$ & $\begin{array}{l}\text { Difficulty } \\
\text { Index }\end{array}$ & Shape No. & Shape Name & $\begin{array}{c}\text { Dots in } \\
\text { Perimeter }\end{array}$ & $\begin{array}{c}\text { Dots in } \\
\text { Area }\end{array}$ & $\begin{array}{l}\text { Difficulty } \\
\text { Index }\end{array}$ \\
\hline 1 & airplane & 236 & 716 & 27.32 & 49 & horseshoe & 273 & 1,310 & 12.99 \\
\hline 2 & alarm clock & 286 & 2,574 & 19.32 & 50 & iron & 226 & 2,039 & 25.70 \\
\hline 3 & angel & 245 & 1,490 & 33.42 & 51 & kangaroo & 213 & 724 & 20.15 \\
\hline 4 & antique chair & 210 & 1,323 & 21.37 & 52 & kerosene lamp & 157 & 879 & 31.38 \\
\hline 5 & baby bottle & 148 & 1,237 & 36.28 & 53 & key & 146 & 840 & 38.96 \\
\hline 6 & banana & 180 & 1,385 & 14.77 & 54 & knife & 133 & 355 & 21.05 \\
\hline 7 & bear & 216 & 1,527 & 26.25 & 55 & leaf & 259 & 1,294 & 15.00 \\
\hline 8 & bell & 155 & 154 & 18.97 & 56 & light bulb & 145 & 1,461 & 17.54 \\
\hline 9 & boot & 185 & 1,709 & 13.08 & 57 & lizard & 202 & 453 & 25.52 \\
\hline 10 & bottle & 143 & 866 & 15.09 & 58 & $\operatorname{man}$ & 249 & 888 & 14.72 \\
\hline 11 & bull & 307 & 1,255 & 25.20 & 59 & man's shoe & 157 & 1,167 & 14.18 \\
\hline 12 & butterfly & 190 & 1,611 & 25.63 & 60 & moth & 257 & 1,642 & 14.22 \\
\hline 13 & camel & 289 & 1,957 & 16.92 & 61 & mushroom & 187 & 1,504 & 13.83 \\
\hline 14 & cap & 160 & 1,524 & 23.29 & 62 & pear & 146 & 1,174 & 16.83 \\
\hline 15 & car & 142 & 820 & 13.70 & 63 & perfume bottle & 204 & 2,034 & 33.16 \\
\hline 16 & cat & 248 & 247 & 26.96 & 64 & piano & 298 & 1,844 & 28.01 \\
\hline 17 & chair & 217 & 1,857 & 28.07 & 65 & pickup & 157 & 783 & 27.47 \\
\hline 18 & chick & 184 & 1,257 & 21.04 & 66 & pig & 223 & 1,363 & 19.10 \\
\hline 19 & coat & 273 & 2,367 & 18.43 & 67 & pipe & 150 & 602 & 21.33 \\
\hline 20 & cow & 254 & 1,480 & 21.25 & 68 & pliers & 225 & 517 & 24.88 \\
\hline 21 & desk lamp & 230 & 592 & 20.83 & 69 & pot & 177 & 1,754 & 22.39 \\
\hline 22 & dinosaur & 263 & 1,100 & 36.43 & 70 & rabbit & 217 & 1,552 & 23.52 \\
\hline 23 & $\operatorname{dog}$ & 291 & 1,527 & 18.47 & 71 & rat & 192 & 785 & 27.52 \\
\hline 24 & doll & 212 & 1,834 & 33.38 & 72 & rhino & 226 & 1,450 & 30.19 \\
\hline 25 & dragonfly & 249 & 1,068 & 22.97 & 73 & rooster & 280 & 1,450 & 21.57 \\
\hline 26 & dress & 199 & 1,519 & 32.32 & 74 & saxophone & 246 & 798 & 21.72 \\
\hline 27 & duck & 172 & 1,025 & 19.99 & 75 & scissors & 250 & 1,186 & 20.68 \\
\hline 28 & dumbbells & 185 & 1,837 & 24.38 & 76 & shorts & 192 & 2,309 & 24.25 \\
\hline 29 & elephant & 271 & 1,766 & 20.97 & 77 & slipper & 139 & 830 & 18.34 \\
\hline 30 & emu & 240 & 1,008 & 13.64 & 78 & snail & 176 & 989 & 19.34 \\
\hline 31 & fire extinguisher & 298 & 1,997 & 21.97 & 79 & snake & 173 & 407 & 29.51 \\
\hline 32 & fire hydrant & 196 & 1,155 & 25.68 & 80 & sock & 144 & 823 & 19.90 \\
\hline 33 & fish & 197 & 1,350 & 20.20 & 81 & spoon & 134 & 416 & 22.02 \\
\hline 34 & flask & 148 & 907 & 24.69 & 82 & spray bottle & 180 & 1,034 & 28.92 \\
\hline 35 & frog & 413 & 2,067 & 23.39 & 83 & starfish & 211 & 1,301 & 11.71 \\
\hline 36 & giraffe & 364 & 1,158 & 12.41 & 84 & table & 289 & 1,357 & 15.89 \\
\hline 37 & glasses & 218 & 651 & 23.77 & 85 & table lamp & 185 & 1,486 & 33.00 \\
\hline 38 & goose & 176 & 930 & 23.68 & 86 & $\operatorname{tank}$ & 186 & 944 & 29.57 \\
\hline 39 & guitar & 155 & 762 & 16.08 & 87 & teddy bear & 238 & 1,571 & 23.84 \\
\hline 40 & gun & 174 & 971 & 30.69 & 88 & telephone & 200 & 2,012 & 21.70 \\
\hline 41 & hammer (ball peen) & 160 & 420 & 30.01 & 89 & toilet & 225 & 2,301 & 27.13 \\
\hline 42 & hammer (claw) & 172 & 505 & 21.25 & 90 & turtle & 171 & 1,100 & 25.07 \\
\hline 43 & hand shovel & 144 & 540 & 27.63 & 91 & vase & 165 & 1,562 & 20.27 \\
\hline 44 & hat & 161 & 1,497 & 21.00 & 92 & violin & 174 & 800 & 16.09 \\
\hline 45 & heart & 171 & 2,686 & 9.12 & 93 & windmill & 243 & 1,330 & 36.77 \\
\hline 46 & helmet & 161 & 1,912 & 30.68 & 94 & wine glass & 234 & 2,091 & 20.61 \\
\hline 47 & hen & 193 & 1,315 & 22.62 & 95 & woman's shoe & 162 & 874 & 17.05 \\
\hline 48 & horse & 290 & 1,118 & 32.47 & 96 & wrench & 159 & 402 & 30.19 \\
\hline
\end{tabular}

Note-The difficulty index specifies the percentage of the full complement of border dots that should be displayed for an expected recognition rate of $75 \%$.

If information from two subsets can be combined before that from the first subset has decayed, this should be sufficient to allow a much higher rate of recognition. Put otherwise, the decline in hit rate as the T3 interval is increased should indicate the size of the integration window for accessing long-term visual memory.

Shape recognition protocol. In Treatment A, with two levels, the subjects were asked to make asked judgments with the room lighted or darkened. Treatment B, with four levels, consisted of the four T3 interval intervals: $0,30,90$, and $270 \mathrm{msec}$. There were, therefore, eight total combinations of the two treatments.

Once a shape has been identified, subsequent recognition is virtually guaranteed, even if the percentage of dots being displayed is reduced or the display conditions are made less accommodating.
Therefore, it was not feasible to display the same shape under each treatment combination. As an alternative, the shapes in the inventory were ranked according to their difficulty indices; then shapes were drawn successively from the rankings and assigned to the eight treatment combinations. To be specific, the random process was restricted by requiring tiered assignment among the eight combinations.

Each of the 10 subjects in Experiment 1 was tested under both lighting conditions, as specified in the Treatment A: Luminance Levels section. Each condition was done as a block; thus, all the shapes requiring a lighted room were tested in one time period, and those requiring a darkened room were tested in another. Order of lighted room or darkened room testing was assigned at random for a given subject. The subjects were required to adapt to the dark for 
A

T1 (Pulse Width) $=0.1 \mathrm{msec}$

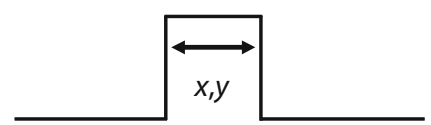

B

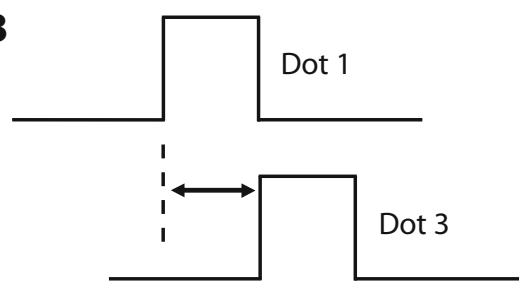

T2 (Pulse Spacing) $=0.1 \mathrm{msec}$

C

T3 (Subset Spacing) $=0,30,90$, or $270 \mathrm{msec}$

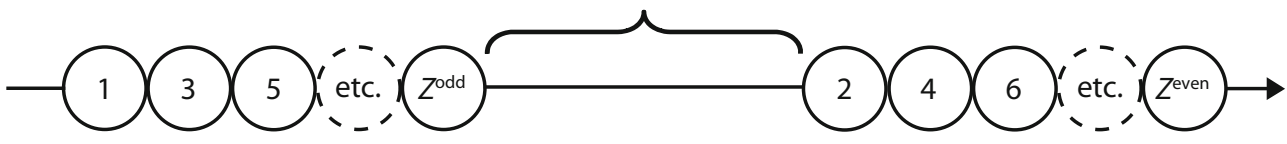

Figure 2. (A) To display a given shape, the address for each dot in the display set was illuminated for 0.1 msec, designated as T1. (B) The set of dots to be displayed was divided into odd and even subsets. Beginning at the starting position (see Figure 1), the odd subset consisted of dots that were at the 1st, 3 rd, 5 th, ... positions. A corresponding enumeration was used for the even subset. T2 specified the onset-to-onset interval between successive pulses in a given subset. $T 2$ was also set at $0.1 \mathrm{msec}$, meaning that there was no delay in the illumination of successive dots within the subset. (C) Here, the pulses are illustrated like beads on a string. For each subset, the last dot is designated as $Z^{\text {odd }}$ and $Z^{\text {even, }}$, respectively, and every dot in the odd subset was shown before any dot in the even subset was shown. The T3 interval was the time between the offset of the odd subset and the onset of the even subset.

30 min before dark room testing, whether that condition came at the beginning of the test session or at the end.

Within a given lighted room or darkened room block, order of display of shapes from the T3 subsets was random. In other words, shapes from all the T3 subsets were picked at random and then were displayed with the $\mathrm{T} 3$ interval that had been assigned to each of the shapes. The subject was asked to name each shape that was displayed, and the experimenter recorded whether an acceptable name had been offered. This was not a speeded task, and response time was not recorded. Guessing was encouraged, and the subjects were not told whether or not their response was correct.

Measuring visible persistence. Before each block of shape testing was conducted, a test for duration of visible persistence was administered. A method was adopted that allowed the visible persistence to be measured using the LED display device, thus assuring comparability with respect to size of the stimuli, wavelength, and control of timing. This method has much in common with the earliest experimental measures of visible persistence, these being done by Segner in 1740 and D'Arcy in 1765 (Allen, 1926). These early studies placed a glowing ember on the rim of a spinning wheel and asked subjects to indicate when the arc being traced by the ember appeared to form an unbroken circle (see also Allport, 1968, and Efron \& Lee, 1971, for more recent related work).

The method used here required rapid display of successive addresses down the column(s) of the LED board. This elicited the perception of a block of simultaneously illuminated dots that moved from the top of the array to the bottom and then began again at the top. Each dot was illuminated for a very brief moment, and the interval between dot activation was varied, as will be detailed below. If that between-dot interval is fairly long, the illuminated block is short, and the height increases as one decreases the interval between activation of successive dots. Rather than being asked for the time interval that would fill the full height of the array - which would be most comparable to asking that a glowing circle be unbroken - the subjects were asked to adjust the timing until the block appeared to be half the full height. This was done to prevent overreaching of the span, for if one has filled the full height of the array, one has minimal basis for knowing whether the interval between pulses has been made too short.

The test called for successively lighting of two adjacent columns of dots that were centered on the display and extended from the top of the array to the bottom. Thus, each column contained 64 dots, for a total of 128 . The dots were activated in sequence as a highly truncated raster scan - that is, Row 1, Column 1; then Row 1, Column 2; then Row 2, Column 1; then Row 2, Column 2; and so forth. Immediately after illuminating the last LED of this array - that is, Row 64, Column 2-the raster sequence began again. (It should be noted that the choice of width of the active array was somewhat arbitrary. One might have chosen to use three columns, or five, or more. Using a height that was the same as the available number [i.e., 64] had the advantage that the edges of the frame provided clear anchor markers for where the columns of the active array began and ended.)

T1 (pulse width) for each dot was fixed at $0.1 \mathrm{msec}$, but T2 (pulse separation, measured onset to onset) was variable. If a long T2 interval was selected, one would see a short group of dots that appeared to move as a block from the top of the display board to the bottom, then returning to the top for another trip. By reducing the T2 interval one could make the block of dots appear taller, and with further reduction, all of the dots in the two columns would appear to be simultaneously illuminated. The $\mathrm{T} 2$ interval was adjusted by the experimenter, under instruction by the subject, till the subject judged the height of the block of moving dots to be half the height of the columns.

For a given visible persistence test, an initial $\mathrm{T} 2$ interval was selected by the computer to lie between 1.0 and $4.0 \mathrm{msec}$. At the upper end of this range, the block of moving dots appeared too short, and at the lower end, it appeared too tall. Clicking of monitor screen buttons increased or decreased T2 in $0.1-\mathrm{msec}$ steps, with the subject saying what direction of change was needed. When the height was deemed to be acceptable - that is, about half the height of the full column - another button-click recorded the judgment. Specifically, what was recorded was the $\mathrm{T} 2$ interval multiplied by 64 , the latter 
being the number of dots that would make up half the height of the two columns. This yielded a value that reflected the number of milliseconds that each pulse was perceived to be visible.

Three successive tests of visible persistence were taken with the room lighted, and three with the room darkened (after full adaptation).

\section{Results}

Differentials of rate of recognition (hit rate). Each shape was presented only once to each subject, displayed as specified by one of the eight treatment combinations ( 2 levels of room illumination $\times 4$ levels of T3 interval). Shapes were randomly assigned to each treatment combination, with approximate matching for difficulty level. The response variable was successful recognition (yes/no).

The appropriate model for this binary data and the experimental design was a generalized linear model (Schall, 1991). Specifically, the design was a mixed model, with subjects and shape being random effects and room illumination and T3 interval being fixed effects. Logit values $\left[\log _{\mathrm{e}}(\right.$ proportion$/ 1$ - proportion)] were calculated, and treatment differences were compared using the standard error of the difference ( $S E D$ ) for these values.

Testing in a darkened room produced significantly more hits than did testing in a lighted room [logit for lighted room $=-0.174$; logit for darkened room $=0.953$; $S E D=0.154 ; p<.001]$.

Order of testing proved to be significant, with testing in the dark first yielding higher hit rates across the room illumination conditions [logit for lighted room first $=$ 0.2067 ; logit for darkened room first $=0.5724$; $S E D=$ $0.1519 ; p<.02]$.

The T3 interval was significant at $p<.001$, but evaluation of this treatment was most meaningful in terms of the differential for lighted and darkened room conditions, as will be discussed below.

Evaluating trends in T3 effect. Arithmetic means for each combination of $\mathrm{T} 3$ interval by room illumination are

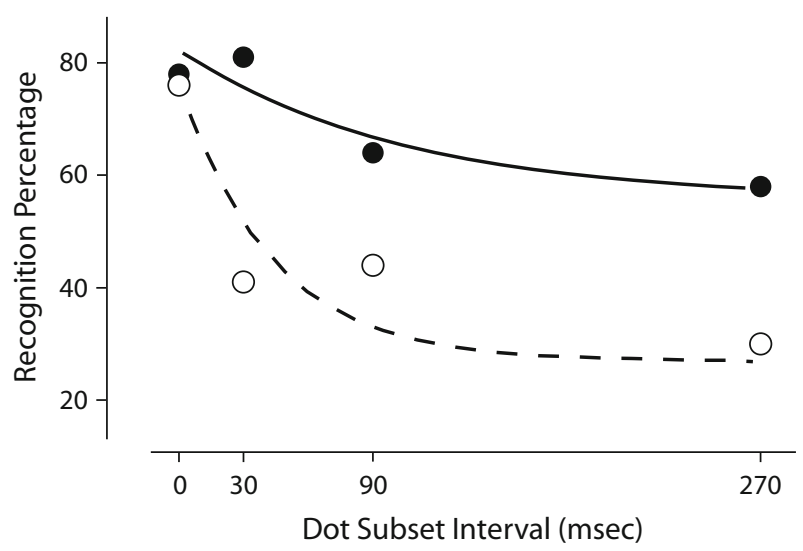

Figure 3. Mean recognition levels for tests done in a darkened room (filled circles) and those done with the room lighted (unfilled circles), plotted for each of the subset intervals in the first experiment. Exponential curves have been added to illustrate trends in the data.
Table 2

Mean Logit Values for Each Treatment Combination (With Standard Errors of the Means) and Model Predictions of Recognition Rates (\%)

\begin{tabular}{|c|c|c|c|c|c|c|}
\hline \multirow{3}{*}{$\begin{array}{c}\mathrm{T} 3 \\
\text { Interval } \\
\text { (msec) }\end{array}$} & \multicolumn{3}{|c|}{ Darkened Room } & \multicolumn{3}{|c|}{ Lighted Room } \\
\hline & \multicolumn{2}{|c|}{ Logit Value } & \multirow[b]{2}{*}{$\%$} & \multicolumn{2}{|c|}{ Logit Value } & \multirow[b]{2}{*}{$\%$} \\
\hline & $M$ & SEM & & $M$ & SEM & \\
\hline 0 & 1.43 & 0.26 & 81 & 1.25 & 0.26 & 78 \\
\hline 30 & 1.51 & 0.28 & 82 & -0.38 & 0.24 & 41 \\
\hline 90 & 0.63 & 0.24 & 65 & -0.40 & 0.24 & 40 \\
\hline 270 & 0.40 & 0.24 & 60 & -0.89 & 0.25 & 27 \\
\hline
\end{tabular}

Note-A generalized linear mixed model was used to transform the percentages for recognition of shapes into logit values - that is, $\log _{e}[$ proportion/( 1 - proportion)]. The logit values have also been backtransformed into model predictions of recognition rates. Note that the predictions of percentages of recognition are very near the arithmetic means that are plotted in Figure 3.

plotted in Figure 3. In this figure, one can see that recognition was comparable for the two room illumination conditions when the T3 interval was $0 \mathrm{msec}$ but that, at other T3 intervals, recognition levels for the lighted room were much lower than they were for the darkened room. The logit values for these recognition rates are given in Table 2, along with the standard errors of the means for those values.

As was indicated above, analysis using the generalized linear mixed model showed that both the overall T3 interval effect (combining both groups) and the difference between the two room illumination conditions were significant at $p<.001$. The interaction, however, was nonsignificant, even though there was a major drop in hit rate from 0 to $30 \mathrm{msec}$ for the lighted room data, but not for the darkened room data. It seemed likely that the similarity of the slopes - which were almost parallel across the other T3 intervals - was obscuring a real differential. Therefore, a second generalized linear mixed model analysis was applied to the lighted room data at these two T3 intervals, which showed the decline of hit rate from 0 to $30 \mathrm{msec}$ to be significant at $p<.001$.

The sharp decline from 0 to $30 \mathrm{msec}$, followed by a much slower decline across the other T3 intervals, is suggestive of an exponential trend. The data were thus reexamined with a nonlinear regression analysis using an exponential model (see Snedecor \& Cochran, 1989). The regressions were extracted successively, asking first about a differential in the mean levels of effect for the two levels of room illumination. This differential proved to be significant at $p<.001$.

Next, the contribution of the linear factor as a function of room illumination was examined, and differentials for lighted versus darkened room conditions were found to be significant at $p<.05$. Finally, the exponential model effects (for lighted vs. darkened room) were found to be significant at $p<.014$.

The modeling that contained all of these contributions was significant at $p<.001$, with the models for lighted and darkened room conditions accounting for $52 \%$ of the variance in the data. Exponential models that show these trends for darkened and lighted room effects have been fitted to the means shown in Figure 3. 
The role of visible persistence. The three visible persistence scores for each subject were averaged for each of the room illumination conditions, and these values had a range of 73-109 with the room lighted and 93-147 in the darkened room. The means across subjects for tests done with the room lighted or darkened were 88 and $122 \mathrm{msec}$, respectively. The standard deviation for the lighted room condition ranged from 3.5 to 13.6 , with a mean of 8.7. For the tests done in the darkened room, the standard deviation of Subject 10 (at 19.3) was deemed to be an outlier. For the other 9 subjects, the range of standard deviations was 4.0-13.1, with the mean being 8.4.

Restricted estimates of maximum likelihood (REML) variance components analysis (using a Wald test for fixed effects) showed the difference in visible persistence duration as a function of room illumination to be significant at $p<.001$.

The key experimental question at issue was whether differences in the duration of visible persistence determines the degree to which information from odd and even subsets can be combined across T3 intervals. Visible persistence is not an issue when $\mathrm{T} 3=0$, because the display of both subsets is virtually simultaneous. If one combines across the other T3 intervals, the mean level of recognition (hit rate) can be plotted against the visible persistence level for each of the 10 subjects, as is shown in Figure 4. The plot clearly displays an effect of room illumination on hit rate, which was confirmed by a REML variance components analysis that yielded a Wald chi square at $p<$ .001 for this factor. However, the association between visible persistence and hit rate was found to be nonsignificant, with $p<.62$.

The formal analysis described in the previous paragraph might not be optimal for determining whether visible persistence contributes to hit rate. To observe a role for visible persistence by means of this analysis, that persistence would have to be seen as independent from room illumi-

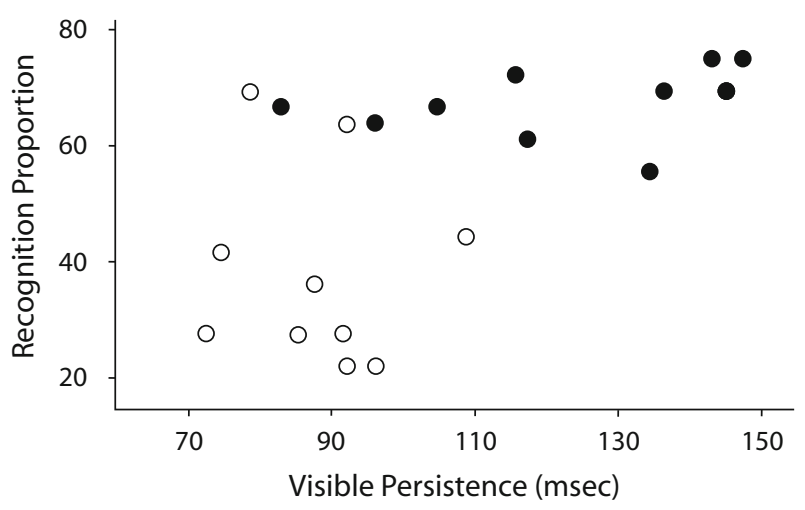

Figure 4. Recognition proportion (hit rate) for each subject under each of the room illumination conditions is plotted against that subject's visible persistence score. Values for $\mathrm{T} 3=0$ were not included in the calculation of the hit rate, since visible persistence would make no contribution to recognition when there is no delay in the presentation of subsets. Although recognition levels were significantly higher in the darkened room (filled circles), the association between visible persistence and hit rate was not significant. nation. However, it is possible that the influence of room illumination acts through visible persistence - that is, having no effect on recognition other than by the process of visible persistence per se. By first looking for differentials of hit rate under the two levels of room illumination, one may have captured effects that should properly be credited to visible persistence, leaving little or nothing to be found when one then asks whether visible persistence also makes a contribution.

On the possibility that the method of analysis would lead to such an inappropriate inference, two additional tests were conducted. First, the REML variance components analysis was run again, using measures of visible persistence as the first factor to be extracted from the data. As was expected, the Wald chi square proved to be significant at $p<.001$. Light was then added as a factor, and this also proved to be significant at $p<.001$. Then the visible persistence measures were dropped from the model, which made a nonsignificant change in the Wald statistic $(p<.78)$.

Second, the contribution of visible persistence to the level of recognition was tested separately for each of the lighting conditions. The duration of visible persistence for the subjects in each subgroup did not predict hit rate with either level of room illumination, with the darkened room probability being .32 and the lighted room probability being .80 .

Further consideration would suggest that the most meaningful test of visible persistence is not to be found in data that combines hit rates across $\mathrm{T} 3$ intervals but in the recognition rate where $\mathrm{T} 3$ has exceeded the duration of visible persistence. Visible persistence levels that were observed for lighted and darkened rooms ( 88 and $122 \mathrm{msec}$ rooms, respectively) should be able to carry information from the first subset across T3 intervals of 30 and $90 \mathrm{msec}$, providing for relatively high hit rates irrespective of room lighting. However, for both levels of room illumination, the durations of visible persistence that were measured would be too short to bridge the 270 -msec T3 interval.

The data plots and models shown in Figure 3 do not match these predictions. For judgments made in a darkened room, hit rates showed only a mild decline over the full range of $\mathrm{T} 3$ interval. There was no major drop at $\mathrm{T} 3=$ 270 , which would be expected because visible persistence in the darkened room averaged only $122 \mathrm{msec}$.

The results for the lighted room judgments are even more telling. Visible persistence lasted an average of $88 \mathrm{msec}$ with this level of room illumination, yet hit rates dropped precipitously and stayed low across all intervals of $30 \mathrm{msec}$ and longer.

Taken together, the evidence from the first experiment does not suggest that the differentials for hit rate can be explained by visible persistence.

\section{EXPERIMENT 2}

\section{Rationale}

The first experiment confirmed that under darkened testing conditions, subjects can integrate visual information- 
for purposes of shape recognition - over a period of a few hundred milliseconds. It is possible that this example of information persistence is the same process that previously has been described as visual information store (Sperling, 1960), iconic memory (Neisser, 1967), or short-term visual storage (Haber \& Standing, 1969).

The first experiment yielded no support for the hypothesis that this information persistence was being mediated by visible persistence. On average, the subjects manifested almost $90 \mathrm{msec}$ of visible persistence in a well-lighted room, but the duration over which the information from odd and even subsets could be integrated was less than $30 \mathrm{msec}$. Testing in a darkened room did greatly increase both the duration over which the information could be integrated and the duration of visible persistence. However, there was substantial integration of the shape cues out to at least $270 \mathrm{msec}$, whereas the average duration of persistence was only $122 \mathrm{msec}$.

Nonetheless, in fairness to the issue, a second experiment was run to further test the possibility that visible persistence serves as the mediator for integration of the shape cues. In this experiment, the subjects were tested under an intermediate (dim) illumination condition. By testing all 96 shapes under the same level of illumination, the statistical tests should be able to better discriminate among the sources of error and determine whether the duration of visible persistence predicts successful recognition. In addition, the $\mathrm{T} 3$ intervals were adjusted to provide more resolution within the interval over which visible persistence is manifested.

\section{Method}

Eight individuals served as subjects for this experiment, each being tested and found to have normal or corrected-to-normal visual acuity. The apparatus, shape stimuli, and testing protocols were the same as for those in Experiment 1, except as follows.

Room illumination was provided by a single $7.5-\mathrm{W}$ bulb that was mounted near the ceiling on the wall behind the subject. This light provided for illumination of the wall on which the display board was mounted of $1 \mathrm{~cd} / \mathrm{m}^{2}$. The duration of visible persistence had been tested using a number of other subjects and several levels of illumination, and this level was found to provide the greatest differences among those individuals. LED emission levels were the same as those for the dark testing in Experiment 1-namely, $9.6 \mathrm{~cd} / \mathrm{m}^{2}$.

Each subject was tested after adapting to the dim illumination for 20 min. The subject was shown all 96 shapes under this level of room illumination, using T3 intervals of 10, 20, 40, and $80 \mathrm{msec}$.

\section{Results}

Mean percentages of recognition across subjects are plotted in Figure 5. There was a linear decline of this hit rate as a function of T3 interval. A generalized linear mixed model analysis confirmed that the decline was significant at $p<.001$, with no sign of a quadratic component $(p=.58)$. Perhaps the decline of recognition in the darkened room (Experiment 1) would have been linear if $\mathrm{T} 3 \mathrm{had}$ been sampled over the shorter range used in the present experiment.

Across the T3 intervals of 10, 20, 40, and $80 \mathrm{msec}$, the logit values - that is, $\log _{\mathrm{e}}$ (proportion $/ 1$ - proportion) were $1.06,1.26,0.26$, and -0.52 , respectively. The maximum and minimum standard errors of means were 0.22 and

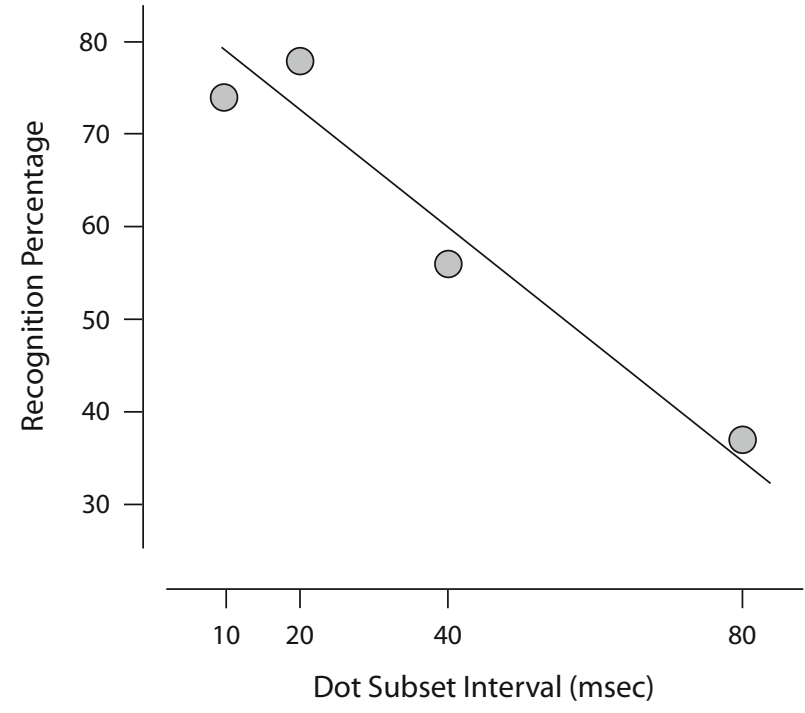

Figure 5. Mean recognition rates (across subjects) for the dim illumination condition in Experiment 2 show a decline across the four subset intervals but no indication of exponential trends. A linear model has been fitted to the data. Note that the range of T3 was much shorter than that used in Experiment 1 (see Figure 3); it is possible that exponential effects are not manifested in this range.

0.19 , with the average being 0.20 . Backtransformed values yielded model predictions of hit rates that were exactly the same as the arithmetic means that are plotted in Figure 5.

Visible persistence scores ranged from 96 to $145 \mathrm{msec}$, with a mean of $111 \mathrm{msec}$. Standard deviations ranged from 0.0 to 7.5 , with a mean of 4.9 .

The relation between visible persistence scores and shape recognition rates was evaluated by linear regression, which did not show the association to be significant ( $p=$ .91 ). Residuals from the model were normally distributed and homogenous. A contingency plot of the two variables is provided in Figure 6.

One could correctly argue that visible persistence should manifest itself more in the slope at which the recognition function declines than in the average of the function summed across T3 intervals. To assess the possibility, a regression analysis was done on the logit-transformed data for each subject, extracting the parameter that reflected the slope of the hit rate across the T3 intervals. Simple linear regression did not fit this sparse data set well, in that residuals were not normally distributed or homogenous. However, robust mixed model regression, which allows for nonnormal residuals and outliers, was able to provide the slope parameters. The association between these slope values and the persistence scores was not significant $(p=.16)$.

The recognition task requires that information be combined - that is, integrated - across T3 intervals. There was a large decline in the success with which the information provided by odd and even subsets could be integrated, even though all the subjects manifested durations of visible persistence that should have been able to bridge the subset interval. Furthermore, the duration of visible persistence 


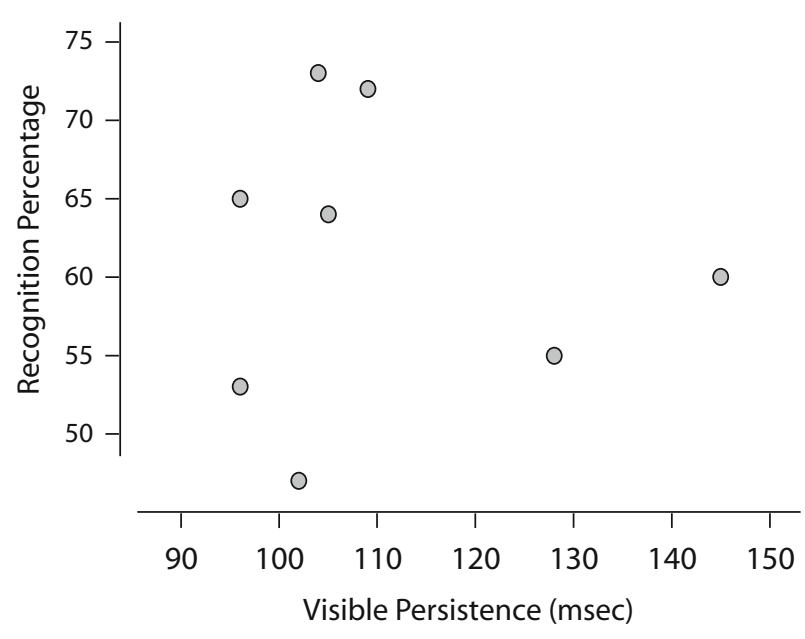

Figure 6. The contingency between recognition rate and visible persistence is plotted for each of the 8 subjects. Here, the percentage of recognition values are the means for each subject, combining across the four subset intervals. The scatterplot does not suggest any relation, which was confirmed by formal analysis (see the Results section).

manifested by a given subject did not predict that subject's hit rate, either in terms of total recognitions or with respect to the slope of the recognition function. Thus, there was nothing in the results of this experiment to suggest that visible persistence mediates integration of information for purposes for object recognition.

\section{DISCUSSION}

The present research measured the duration of information persistence that allows integration of partial shape cues over time and examined whether this process is mediated by conscious visible persistence. The results show large differentials of duration of information persistence as a function of room illumination and indicate that the information persistence needed for integration of minimal transient shape cues is not based on visible persistence. Before this evidence is discussed, a little background should be given about the measure of visible persistence that was used.

As has been noted by Allen (1926), it can hardly be doubted that from the earliest ages, when in the dark, one would perceive the stimulus from an ember at the tip of a revolving stick as a circle of light. In Query 16, Newton $(1730 / 1952)$ was one of the first to articulate that this perceptual phenomenon may be due to sustained activation of what we now know to be the retina: "And when a Coal of Fire moved nimbly in the circumference of a Circle, makes the whole circumference appear like a Circle of Fire: Is it not because the Motions excited in the bottom of the Eye by the Rays of Light are of a lasting nature, and continue till the Coal of Fire in going round returns to its former place?" (p. 347).

Formal measures of visible persistence, using the method suggested by Newton, were first done by Segner in 1740 , followed by D'Arcy in 1765, who apparently was unaware of the earlier work (Allen, 1926). The D'Arcy apparatus consisted of a rapidly rotating wheel whose speed could be regulated and measured. A glowing coal was placed on the rim of the wheel, and D'Arcy judged the speed that was needed for the arc of light to be seen as an unbroken circle. Observations were made at night, and he measured the duration of the visible persistence to be eight "tierces," which converts to $133 \mathrm{msec}$. It is interesting that D'Arcy's measure, taken almost 3 centuries ago, is very close to the mean value of $122 \mathrm{msec}$ that was found here when the room was dark.

As was outlined in the introduction, Di Lollo and Bischof (1995) provided convincing arguments that the duration of visible persistence is heavily influenced by the level of ambient room illumination. In keeping with their predictions and with the numerous studies that they reviewed, the present experiments showed that the duration of visible persistence was greater when illumination was toward the scotopic end of the range. In Experiment 1, the use of a lower stimulus intensity for dark room testing may have contributed to the differential (Bowen et al., 1974; Efron, 1970; Hansteen, 1971).

With respect to information persistence, however, the present results do not support the general assumption that it is mediated by visible persistence. Each of the shapes to be identified was rendered as a set of minimal cues, and these were further divided into subsets, which, if presented alone, would not be sufficient to trigger recognition. In order to identify a given object, the subject had to integrate shape cue information across the interval that separated the subsets, requiring persistence of the information provided by these cues. This information persistence was heavily influenced by room illumination, being longer when the room was dark than when it was bright, but it does not appear that this effect was mediated by visible persistence.

In Experiment 1, recognition levels in the darkened room declined only moderately-from about $80 \%$ to $60 \%$ - as the subset interval was increased to $270 \mathrm{msec}$. The duration of visible persistence was only $122 \mathrm{msec}$, on average, with the longest durations being no more than $150 \mathrm{msec}$. If visible persistence were mediating the transfer of information across the subset interval, the hit rate should have dropped precipitously between the 90- and the 270-msec subset intervals, but this did not occur.

Conversely, in Experiment 1, recognition performance with the room bright dropped from about an $80 \%$ hit rate at the $0-\mathrm{msec}$ interval to $40 \%$ at $30 \mathrm{msec}$, even though visible persistence lasted for almost $90 \mathrm{msec}$. If visible persistence were mediating information transfer across the subset interval, one would have expected higher hit rates for subset intervals into the 90-msec range.

Finally, neither experiment showed individual visible persistence durations to be predictive of recognition performance of these individuals. Taken together, the evidence suggests that the ability to integrate subminimal shape cues for purposes of recognition requires some form of information persistence that is not mediated by the duration over which a brief stimulus appears to be visible.

Haber and Standing (1969) were very explicit in equating visible persistence (as studied by synchrony of judg- 
ment) with information persistence (as studied by partial report). Turvey (1978) and Sakitt (1975; 1976a, 1976b) took similar views.

Coltheart (1980), however, thoroughly examined the evidence that would distinguish information persistence from the poststimulus perceptual state of visible persistence and included the cue integration studies of Eriksen and Collins $(1967,1968)$ as an example of information persistence. Coltheart proposed that stimulus conditions that would affect the duration of visible persistence should not have an influence on information persistence.

The present results support the analysis of Coltheart (1980). Room illumination is a major factor that affects the duration of visible persistence, as detailed by Di Lollo and Bischof (1995), and both of the present experiments showed that the information persistence required for integration of partial shape cues is affected by the level of illumination. Although visible persistence did not mediate the information persistence required for cue integration, both showed inverse-intensity effects. This suggests similarity of perceptual (and neural) response to differences in illumination, which most likely is determined by the degree to which one is working at or near the scotopic range, as was suggested by Di Lollo and Bischof (1995).

Di Lollo and Bischof (1995) proposed that one will not see direct-intensity effects if there is no difference in the salience of the stimulus. Their analysis provided a major reason to adjust the brightness of the stimulus for dark room testing to be lower than that for bright room testing. The stimulus intensities that were used succeeded in producing approximately equal hit rates for $\mathrm{T} 3=0$ - that is, when there was no delay in the display of successive subsets. In keeping with their prediction, there were no direct-intensity effects. On the other hand, the information persistence required for shape cue integration showed clear inverse-intensity effects, a result not predicted by Di Lollo and Bischof.

It is well understood that the visual system dramatically increases its level of sensitivity when faced with low-light conditions, and this also causes an increase in the interval over which stimulation is integrated (Barlow, 1958; Savage, 1996; Warrant, 1999). Signal-to-noise ratio is doubtless a factor (Hemila, Leber, \& Donner, 1998; Tjan, Braje, Legge, \& Kersten, 1995). For shape recognition, any information being delivered from the background - for example, the contours of the display frame or the array of nonemitting LEDs - represents noise with respect to the processing of shape cues. The amount of noise decreases as the lighting dims, so it would be reasonable that shape cues could be successfully integrated over a longer duration. The window for integration might be a function only of relative luminance - that is, the ratio of stimulus luminance to background luminance - but likely the "noise" from background contours and patterns influences the duration over which shape cues can be integrated.

Most studies of information persistence, especially those that have been offered under the heading of iconic memory, have shown a decay function in the 100 - to 300 msec range. For the present tests that were done in the dark, the performance was well above chance at $260 \mathrm{msec}$, and the slope was so shallow that above-chance performance would have been likely if much longer intervals had been used. This might invite speculation that the integration of cues was being mediated by a process having an intrinsically longer time course, such as visual shortterm memory. Brockmole, Wang, and Irwin (2002), for example, used a missing dot protocol (Di Lollo, 1980) to demonstrate that information from the initial subset does not decay and, instead, provides progressively more information at interstimulus intervals ranging upward of $5,000 \mathrm{msec}$. These authors argued that the information is being carried forward in visual short-term memory and, thus, is available to assist performance long after information persistence (e.g., iconic memory) has faded away.

The two experiments reported here provide only a limited basis for inference, but on the face of it, they would appear to suggest a single process that allows for integration of the information contained in the two subsets as a function of light level. With a bright room, the duration over which the subsets can be integrated drops rapidly; in a dim room, it drops more slowly; and in a dark room, more slowly yet. It is possible that one is transitioning from a short-duration information persistence to a longer duration short-term memory process, but support of that interpretation will have to wait for additional evidence.

\section{AUTHOR NOTE}

This research was supported, in part, by the Quest for Truth Foundation. Ronald Henry and Andrew Jones provided measures of ambient and LED light levels, respectively. Statistical analysis was provided by Leigh Callinan. Custom software and firmware was written by David Gorin at Dark Horse Software. Correspondence concerning this article should be addressed to E. Greene, Laboratory for Neurometric Research, Department of Psychology, University of Southern California, Los Angeles, CA 90089-1061 (e-mail: egreene@usc.edu).

\section{REFERENCES}

Adrian, E. D., \& Matthews, R. (1927). The action of light on the eye: Pt. I. The discharge of impulses in the optic nerve and its relation in the electric changes in the retina. Journal of Physiology, 63, 378-414.

Allan, L. G. (1979). The perception of time. Perception \& Psychophysics, 26, 340-354.

Allen, F. (1926). The persistence of vision. American Journal of Physiological Optics, 7, 439-457.

Allport, D. A. (1968). Phenomenal simultaneity and the perceptual moment hypothesis. British Journal of Psychology, 59, 395-406.

Averback, E., \& Coriell, E. (1961). Short-term memory in vision. Bell System Technical Journal, 40, 309-328.

BARLOW, H. B. (1958). Temporal and spatial summation in human vision at different background intensities. Journal of Physiology, 141, 337-350.

Bowen, R. W., Pola, J., \& Matin, L. (1974). Visual persistence: Effects of flash luminance, duration and energy. Vision Research, 14, 295-303.

Brockmole, J. R., Wang, R. F., \& Irwin, D. E. (2002). Temporal integration between visual image and visual percepts. Journal of Experimental Psychology: Human Perception \& Performance, 28, 315-334.

Coltheart, M. (1980). Iconic memory and visible persistence. Perception \& Psychophysics, 27, 183-228.

Di Lollo, V. (1977). Temporal characteristics of iconic memory. $\mathrm{Na}$ ture, 267, 241-243.

Di Lollo, V. (1980). Temporal integration in visual memory. Journal of Experimental Psychology: General, 109, 75-97.

Di Lollo, V., \& BischoF, W. F. (1995). Inverse-intensity effect in duration of visible persistence. Psychological Bulletin, 118, 223-237. 
Di Lollo, V., Enns, J. T., Yantis, S., \& Dechief, L. G. (2000). Response latencies to the onset and offset of visual stimuli. Perception \& Psychophysics, 62, 218-225.

Di Lollo, V., Seiffert, A. E., Burchett, G., Rabeeh, R., \& Ruman, T. A. (1997). Phosphor persistence of oscilloscopic displays: A comparison of four phosphors. Spatial Vision, 10, 353-360.

Di Lollo, V., \& Woods, E. (1981). Duration of visible persistence in relation to range of spatial frequencies. Journal of Experimental Psychology: Human Perception \& Performance, 7, 754-769.

EFron, R. (1970). Effect of stimulus duration on perceptual onset and offset latencies. Perception \& Psychophysics, 8, 231-234.

EFron, R., \& LeE, D. N. (1971). The visual persistence of a moving stroboscopically illuminated object. American Journal of Psychology, 84, 365-375.

Eriksen, C. W., \& Collins, J. F. (1967). Some temporal characteristics of visual pattern perception. Journal of Experimental Psychology, 74, 476-484.

ERIKSEN, C. W., \& Collins, J. F. (1968). Sensory traces versus the psychological moment in the temporal organization of form. Journal of Experimental Psychology, 77, 376-380.

Haber R. N., \& STANDING, L. (1969). Direct measures of short-term visual storage. Quarterly Journal of Experimental Psychology, 21, 43-54.

HANSTEEN, R. W. (1971). Visual latency as a function of stimulus onset, offset, and background luminance. Journal of the Optical Society of America, 61, 1190-1195.

Hawkins, H. L., \& Shulman, G. L. (1979). Two definitions of persistence in visual perception. Perception \& Psychophysics, 25, 348-350.

Hemila, S., Lerber, T., \& Donner, K. (1998). Noise-equivalent and signal-equivalent visual summation of quantal events in space and time. Visual Neuroscience, 15, 731-742.

Hogben, J. H., \& Di Lollo, V. (1974). Perceptual integration and perceptual segregation of brief visual stimuli. Vision Research, 14, 1059-1069.

IRWIN, D. E., Yantis, S., \& Jonides, J. (1983). Evidence against visual integration across saccadic eye movements. Perception \& Psychophysics, 34, 49-57.

Irwin, D. E., \& Yeomans, J. M. (1986). Persisting arguments about visual persistence: Reply to Long. Perception \& Psychophysics, 39, 225-230.

Jonides, J., IRwin, D. E., \& YANTIS, S. (1982). Integrating visual information from successive fixations. Science, 215, 192-194.

Jonides, J., IRWIN, D. E., \& YANTIS, S. (1983). Failure to integrate information from successive fixations. Science, 222, 188.

Keele, S. W., \& Chase, W. G. (1967). Short-term visual storage. Perception \& Psychophysics, 2, 383-386.

LoNG, G. M. (1979). Comment on Hawkins and Shulman's Type I and Type II visual persistence. Perception \& Psychophysics, 26, 412-414.

LoNG, G. M. (1980). Iconic memory: A review and critique of the study of short-term visual storage. Psychological Bulletin, 88, 785-820.

LONG, G. M., \& GILDEA, T. J. (1981). Latency for the perceived offset of brief target gratings. Vision Research, 21, 1395-1399.
LONG, G. M., \& McCARThY, P. R. (1982). Rod persistence on a partialreport task with scotopic and photopic backgrounds. American Journal of Psychology, 95, 302-322.

LoNG, G. M., \& SAKITT, B. (1980). The retinal basis of iconic memory: Eriksen and Collins revisited. American Journal of Psychology, 93, 195-207.

NeIsser, U. (1967). Cognitive psychology. New York: AppletonCentury-Crofts.

Newton, I. (1952). Opticks. New York: Dover. (Original work published 1730)

NisLY, S. J., \& WASSERMAN, G. S. (1989). Intensity dependence of perceived duration: Data, theories, and neural integration. Psychological Bulletin, 106, 483-496.

Phillips, W. A. (1974). On the distinction between sensory storage and short-term visual memory. Perception \& Psychophysics, 16, 283-290.

Rayner, K., \& Pollatsek, A. (1983). Is visual information integrated across saccades? Perception \& Psychophysics, 34, 39-48.

SAKITT, B. (1975). Locus of short-term visual storage. Science, $\mathbf{1 9 0}$ 1318-1319.

Sakitt, B. (1976a). Iconic memory. Psychological Bulletin, 83, 257-276.

SAKITT, B. (1976b). Psychophysical correlates of photoreceptor activity. Vision Research, 16, 129-140.

SAKITT, B., \& Long, G. M. (1978). Relative rod and cone contributions in iconic storage. Perception \& Psychophysics, 23, 527-536.

SAKITT, B., \& LonG, G. M. (1979). Spare the rod and spoil the icon. Journal of Experimental Psychology: Human Perception \& Performance, 5, 19-30.

SaVAGE, G. L. (1996). Temporal summation for grating patches detected at low light levels. Optometry \& Vision Science, 73, 404-412.

SCHALL, R. (1991). Estimation in generalized linear models with random effects. Biometrika, 40, 917-927.

Snedecor, G. W., \& Cochran, W. G. (1989). Statistical methods (8th ed.). Ames: Iowa State University Press.

SPERLING, G. (1960). The information available in brief visual presentations. Psychological Monographs, 74, 1-29.

Tuan, B. S., Braje, W. L., Legge, G. E., \& Kersten, D. (1995). Human efficiency for recognizing 3-D objects in luminance noise. Vision Research, 35, 3053-3069.

Turvey, M. T. (1978). Visual processing and short-term memory. In W. K. Estes (Ed.), Handbook of learning and cognitive processes (Vol. 5, pp. 91-142). Hillsdale, NJ: Erlbaum.

WARrant, E. J. (1999). Seeing better at night: Life style, eye design and the optimum strategy of spatial and temporal summation. Vision Research, 39, 1611-1630.

(Manuscript received February 7, 2006; revision accepted for publication November 30, 2006.) 